\title{
Quiescence Detection in a Distributed KLIC Implementation
}

\author{
Kazuaki Rokusawa ${ }^{\star}$ Akihiko Nakase ${ }^{\star \star}$ Takashi Chikayama $^{\star \star \star}$ \\ \{rokusawa, nakase, chikayama\}@icot.or.jp \\ Institute for New Generation Computer Technology \\ 2-3-3, Shiba, Minato-ku, Tokyo 105, JAPAN
}

\begin{abstract}
Quiescence detection is a fundamental facility for parallel and distributed processing. This paper describes schemes for quiescence detection in a distributed KLIC implementation. KLIC is a portable implementation of concurrent logic programming language KL1. Termination is detected using the weighted throw counting (WTC) scheme. Based on the scheme a scheme for global suspension was invented. The postmortem system built-in predicate which provides meta programming facilities was designed, and its distributed implementation is also presented.
\end{abstract}

Keywords: concurrent logic programming, termination detection, quiescence detection, distributed implementation, weighted reference counting

\section{Introduction}

This paper describes schemes for quiescence detection (termination and global suspension detection) in a distributed KLIC implementation. KLIC [1] is a portable implementation of concurrent logic programming language KL1 [2] which compiles into $\mathrm{C}$ code.

Quiescence detection is fundamental to parallel and distributed processing. It is not easy to detect quiescence for distributed computation because of the difficulty in obtaining a consistent global state. Although some parallel programs can detect termination by itself, for example, using the short circuit technique, extra computation is usually needed; the same number of extra unifications which close switches as generated processes are required in general. In addition, detection may be incorrect because of bugs. Deadlock or perpetual suspension occurs frequently in developing parallel programs, which is hard to find and fix, especially when processes are distributed among processors. Therefore, parallel programming systems themselves should provide global suspension detection.

Considering the above, we adopted quiescence detection in the distributed KLIC implementation. Termination is detected using the weighted throw counting

\footnotetext{
* R \& D Group, Oki Electric Industry Co., Ltd., since April 1, 1995

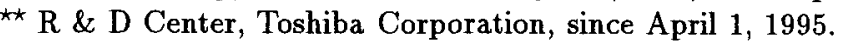

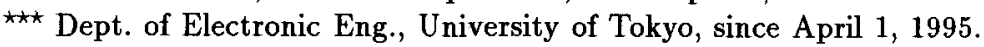




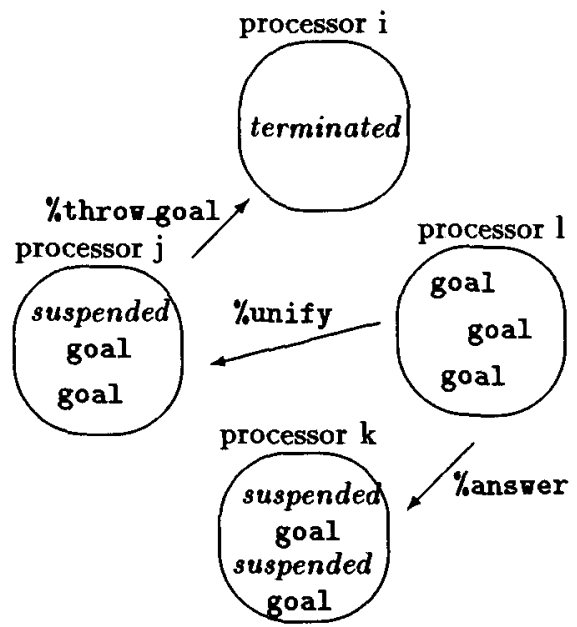

(1) Messages are in transit.

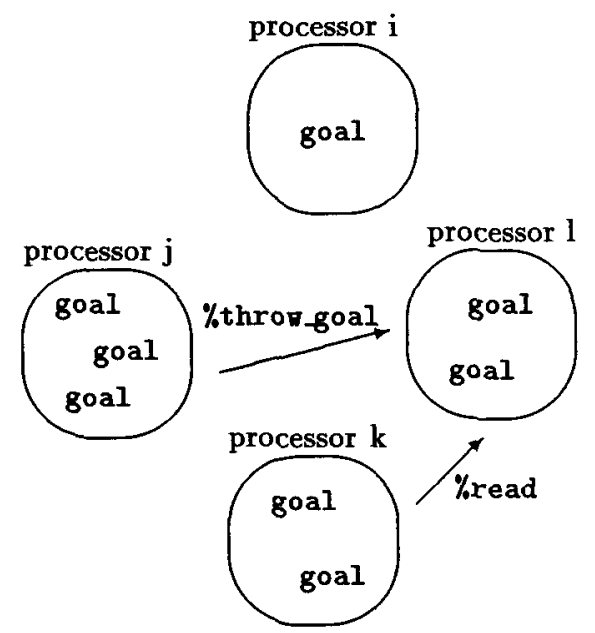

(2) Processors $\mathbf{i}$ and $\mathbf{k}$ are activated.

Fig. 1. Execution behavior in distributed environments.

(WTC) scheme [3], which has been employed for several years in previous KL1 implementations on Multi-PSI and PIMs [4, 5]. A scheme for global suspension detection based on the WTC scheme was invented.

We also designed the postmortem system built-in predicate, which provides programmers with meta programming facilities. An implementation of the predicate for distributed environments was also invented.

This paper is organized as follows. Section 2 describes execution behavior of KL1 programs and quiescence detection problems in distributed environments. Section 3 presents schemes for termination detection and global suspension. Experimental studies of the WTC scheme for termination detection on a PIM/m machine are given in section 4 . Section 5 describes the design and distributed implementation of the postmortem predicate.

\section{Computation Model}

This section gives a sketch of the execution behavior of KL1 programs in distributed environments, and defines the quiescence detection problems.

\subsection{The KL1 Language}

KL1 is a concurrent logic programming language based on GHC (Guarded Horn Clauses) [6]. Its basic execution mechanism is common with other languages of the family, such as Concurrent Prolog [7], Parlog [8] or Janus [9]. 
Unlike GHC which is a theoretical language, KL1 is designed as a practical language for writing an operating system and application programs to execute on parallel computers. It also provides primitives for load distribution, which is done by means of pragma attached to goals of the form goal@node(Proc). A goal with the pragma is thrown to specified processor. Note that the semantics of programs with pragmas are the same as with the pragmas removed.

\subsection{Execution Behavior}

In distributed environments, goals are distributed over processors. Each processor executes goal reductions independently. The reduction may succeed, suspend, or fail. In the first case, the goals with pragmas are thrown out to other processors.

Throwing of a goal is done by sending a \%throw goal message ${ }^{4}$, which carries some identifier and encoded arguments relating to the goal. A processor may also send a message in order to perform distributed unification, remote value fetch, and distributed garbage collection. Messages carry a unify request (\%unify), a read request (\%read), and a dereferenced result (\%answer), etc ${ }^{5}$. Since messages are delivered with an arbitrary finite delay, there may be messages in transit at a given time. Figure 1 shows the execution behavior in distributed environments.

\subsection{Definition of the Problems}

A processor is locally terminated when all goals in it terminate and no suspended goals reside in it. If some goals are suspended, but no active goals reside, the processor is locally suspended. We define the following stable states as termination and global suspension respectively.

Termination: All processors are locally terminated, and no messages are in transit.

Global suspension: All processors are either locally terminated or suspended, and no messages are in transit. At least one processor is locally suspended. This state includes deadlock and perpetual suspension.

\section{Quiescence Detection}

This section first describes why quiescence detection is difficult in a distributed KLIC implementation. After that, schemes for termination detection and global suspension detection are presented.

\footnotetext{
In this paper, message names are marked by prefixing them with a percent sign.

${ }^{5}$ Details are described in $[10,11]$.
} 


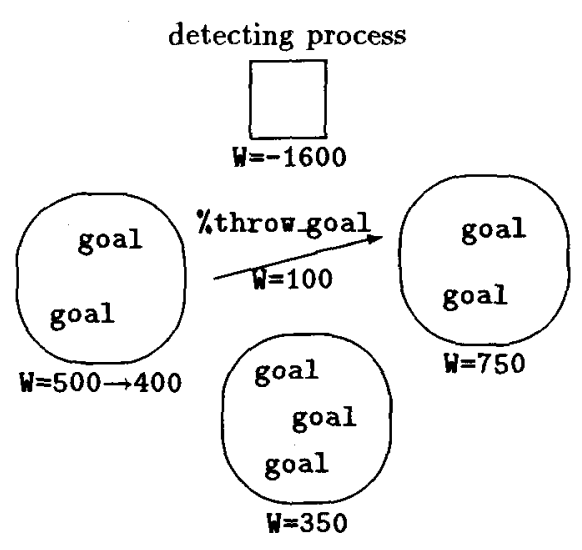

(1) Sending a message

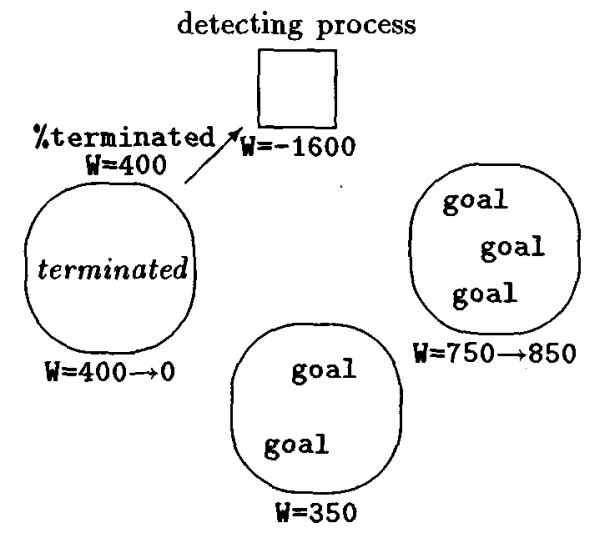

(2) Receiving a message and local termination

Fig. 2. Snapshots under the WTC scheme.

\subsection{Difficulty}

Unlike sequential computation, quiescence detection is not trivial for distributed computation because of the difficulty in obtaining a consistent global state.

Since messages may be in transit at a given time, even when each processor is in a stable state, it does not mean that the whole computation has become globally stable. There may be such messages in transit that carry goals, or bring dereferenced results and wake up suspended goals. In figure 1 (1), processor $i$ and $k$ are stable; processor $i$ is locally terminated and processor $k$ is suspended. However, both became active in figure 1 (2) after receiving messages; processor $i$ receives a \%throw goal message from processor $j$, and processor $k$ resumes suspended goals after receiving an \%answer message from processor $\mathrm{l}$.

\subsection{Termination Detection}

We present here a scheme for termination detection called the weighted throw counting (WTC) scheme [3]. The scheme is an application of the weighted reference counting (WRC) scheme $[12,13]$, which is a garbage collection scheme for parallel processing systems ${ }^{6}$. In previous KL1 implementations on Multi-PSI and PIMs, the WTC scheme has been employed to detect termination.

\section{The WTC Scheme}

A detecting process is placed on one of the processors to actually detect termination. The WTC scheme associates some weight to the detecting process, each

\footnotetext{
${ }^{6}$ Derivation of termination detection from the WRC scheme is described in [14].
} 
processor, and each message in transit. The weight of a message in transit and that of a processor are positive integers, while the weight of the detecting process is a negative integer. The WTC scheme maintains the invariant that:

The sum of the weights is zero.

This ensures that the weight of the detecting process reaches zero if and only if all processors locally terminate and no messages are in transit ${ }^{7}$.

When a processor sends a message, it assigns a weight to the message and subtracts the same amount from its weight. The new weight of the processor and that assigned to the message should both be positive, and the sum of the two weights is equal to the original weight of the processor. When a processor receives a message, it adds the weight of the message to its (positive) weight.

When a processor locally terminates, it sends a \%terminated message to the detecting process to return its weight. On receiving a \%terminated message, the detecting process adds the weight carried to its (negative) weight. If the weight of the detecting process reaches zero, a global termination is detected. Figure 2 shows snapshots under the WTC scheme.

\section{When the Weight Becomes One}

When the weight of a processor becomes one, the processor cannot send a message, because the weight cannot be divided.

In this case, the processor sends a \%request message requesting more weight to the detecting process. Message sending is suspended until the weight becomes more than one. On receiving a \%request message, the detecting process sends back a \%supply message which carries a large amount of weight to the sender processor. When a processor receives a \%supply message from the detecting process or some other message with some weight from another processor, it adds the weight to its weight, which enables it to send any suspended messages.

\section{How to Assign a Weight}

We discuss here the strategy to assign a weight which decreases the number of requesting weight. In the worst case, a \%request message is sent on each message sending, while no \%request message is sent in the best case.

- A \%throw goal message should bring some large amount of weight, because it may reach a locally terminated processor which has no weight. The reactivated processor may send messages, which requires some amount of weight.

- Messages carrying a value (\%answer and \%unify) need not have a large amount of weight. Although a locally suspended processor may receive those messages and it may resume message sending, the weight of the processor is expected to be large enough. When a locally terminated processor receives those messages, no more computation will begin.

${ }^{7}$ Essentially the same scheme named the Credit Distribution and Recovery algorithm [15] was independently invented after the WTC scheme was. Credit corresponds to weight in the WTC scheme. 
- Only a small amount of weight is enough for a read request message (\%read), because it brings no computation to the destination processor.

- A \%supply message should carry a very large amount of weight. Although the weight assigned to a message sent by a processor must be less than the weight of the processor, the weight carried by \%supply does not have this limitation, because a \%supply message is sent by the detecting process.

Considering above, we adopted the following strategy.

- Assign a fixed large amount of weight (say $2^{12}$ ) to a \%throw-goal message if the weight of the processor is more than twice of that; otherwise assign half of the weight of the processor.

- Assign a fixed small amount of weight (say 10) to \%unify, \%read, and \%answer messages if the weight of the processor is more than twice of that; otherwise assign half of the weight of the processor.

- Assign a very large amount of weight (say $2^{24}$ ) to a \%supply message.

Once a processor receives a \%supply message, it can send a message at least $2^{12}$ times without receiving any weight.

\subsection{Global Suspension Detection}

In previous implementations on Multi-PSI and PIMs, perpetual suspension within a processor could be detected using MRB $[16,17]$ and local garbage collection [18]. However, they had no function for distributed environments.

We describe here a basic concept and two schemes for global suspension detection. Both the two schemes are based on the WTC scheme.

\section{Basic Concept}

In the state of global suspension, each processor is either locally suspended or locally terminated and no messages are in transit. Since a terminated processor has no weights, the sum of the weights of the detecting process and the suspended processor becomes zero, which can be used to detect global suspension.

\section{Returning Weight Scheme}

If a locally suspended processor returns its weight, global suspension can be detected in the same way as termination detection.

When a processor becomes locally suspended, it also returns its weight by sending a \%suspended message to the detecting process. When the weight of the detecting process reaches zero, either termination or global suspension is guaranteed. This is because a locally suspended processor can be reactivated and can terminate at any time. If the communication channels are FIFO, when the last message is \%suspended, global suspension is guaranteed. However, if the channels are non-FIFO, or, the last message is \%terminated, no decision can 


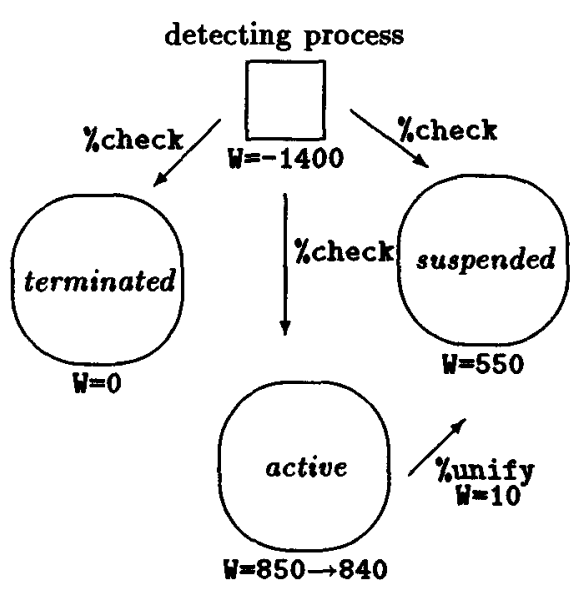

(1) Broadcasting a \%check message

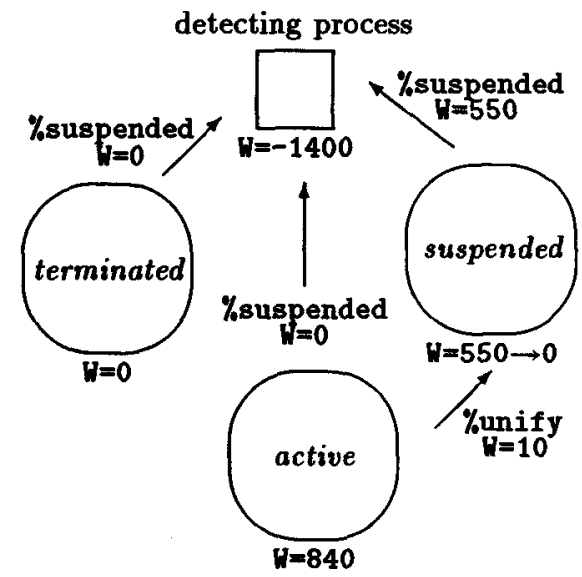

(2) Sending back a \%suspended message

Fig. 3. Global suspension detection using the collecting weights scheme.

be made. To decide between the two states, the detecting process broadcasts a message asking for a report of the state. If the detecting process receives a report of termination from all processors, termination is guaranteed. Otherwise, global suspension is detected.

Since this scheme requires no originator, the delay of detection can be minimaized. However, as the weight of a processor is returned back on each local suspension, the weight of a processor may become one frequently, and frequent \%request/\%supply messages may degrade the performance. Therefore, this scheme must be effective in a debugging phase where high performance is not required, but frequent global suspension is expected.

\section{Collecting Weights Scheme}

We present here a scheme where the detecting process collects the weights of suspended computation, and decides whether global suspension occurs or not.

On start of detection, the detecting process allocates the ack counter, and sets the initial value at the number of processors. The detecting process then broadcasts a \%check message which asks for a return of the weight of suspended computation (figure $3(1)$ ).

On receiving a \%check message, if the processor is locally suspended, it sends back a \%suspended message which carries all its weights. Otherwise, that is, when the processor is active (it has some active goals) or locally terminated, it sends back a \%suspended message with no weight (figure 3 (2)). 
Table 1. Execution results on PIM/m.

\begin{tabular}{|c|c|c|c|c|c|c|}
\hline \multirow[t]{2}{*}{ Programs } & \multirow{2}{*}{\multicolumn{2}{|c|}{$\begin{array}{c}\text { execution } \\
\text { time (sec) reductions }\end{array}$}} & \multicolumn{4}{|c|}{ number of messages } \\
\hline & & & $\%$ throw \%unify & \%term & \%req & \%sup \\
\hline \multirow[t]{2}{*}{ MGTP } & 1,986 & $6,916 \mathrm{M}$ & 549,841 & 47 & 36 & 36 \\
\hline & 348 & $7,432 \mathrm{M}$ & 639,856 & 306 & 281 & 281 \\
\hline \multirow[t]{2}{*}{ Gene } & 5,457 & $12,962 \mathrm{M}$ & 8,670 & 1,056 & 1,054 & 1,054 \\
\hline & 793 & $12,962 \mathrm{M}$ & 8,670 & 8,642 & 8,6708 & $8,6 \overline{70}$ \\
\hline $64 \mathrm{PEs}$ & 104 & $32 \mathrm{M}$ & 236,540 & 449 & 447 & 447 \\
\hline \multirow{3}{*}{$\frac{\text { LogSim }}{\text { DisPool }}$} & 356 & $983 \mathrm{M}$ & $2,5548,219,263$ & 257 & 255 & 255 \\
\hline & 53 & $26 \mathrm{M}$ & $1,030 \quad 32,222$ & 441 & 339 & 339 \\
\hline & 249 & $231 \mathrm{M}$ & $7,750 \quad 1,972,286$ & 2,685 & $\overline{2,5832}$ & 2,583 \\
\hline \multirow[t]{2}{*}{ Pentomino 32} & 665 & $1,974 \mathrm{M}$ & $13,639 \quad 20,933$ & 389 & 387 & 387 \\
\hline & 134 & $1,974 \mathrm{M}$ & $56,8 \overline{72}$ & 4,445 & 4,443 & 4,443 \\
\hline COMPILE $21 \mathrm{PEs}$ & 282 & $43 \mathrm{M}$ & 7,150 & 354 & 228 & 228 \\
\hline
\end{tabular}

On receiving a \%suspended message, the detecting process adds the received weight to its (negative) weight, and decrements the ack counter.

When both the weight of the detecting process and the ack counter reach zero, either termination or global suspension is guaranteed. The decision between the two states can be made by the same operations as those in the returning weight scheme. If the value of the ack counter only reaches zero, it is guaranteed that some processors are active, or some messages remain in transit.

Unlike the returning weight scheme, this scheme requires an originator which demands the detecting process to start detection operations. Although when and how to originate the detection operations depends on each application, the repeated observation at regular intervals (for example ten senconds) must be applicable to many cases.

\section{Experimental Studies of the WTC Scheme on PIM/m}

As mentioned before, the WTC scheme has been employed for several years in KL1 implementations on the Multi-PSI and PIMs. We evaluate here the effectiveness of the WTC scheme for termination detection through the experience on a PIM $/ \mathrm{m}$ machine, which is one of the five models of PIM.

\section{Execution Results}

Table 1 shows execution times, reductions, and the number of messages related to termination detection for several programs ${ }^{8}$. The following are brief explanation of the programs.

\footnotetext{
${ }^{8}$ Messages for remote value fetch and garbage collection are not concerned.
} 
Table 2. Additional messages of the two schemes.

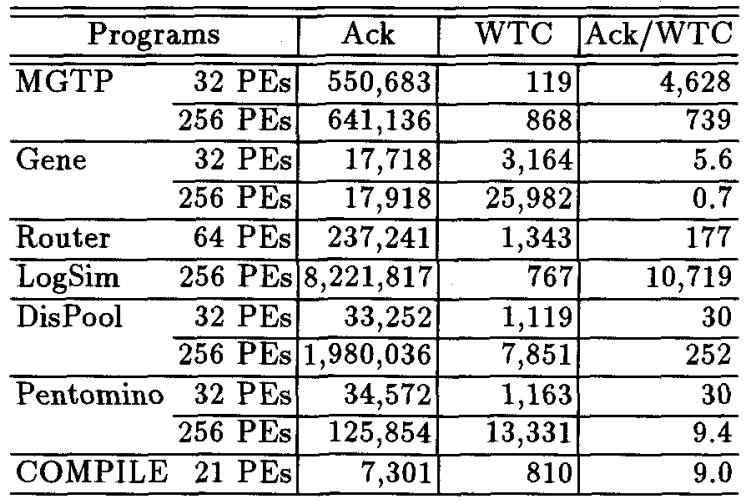

MGTP: A parallel model-generation based theorem prover. It exploits ORparallelism for non-Horn problems and AND-parallelism for Horn problems [19]. Open problems in finite algebra have been proved using MGTP [20].

Gene: A parallel application program for genetic sequence analysis based on an iterative improvement technique [21].

Router: A LSI routing program based on a concurrent objects model. Each line segment is represented as a object, and routing paths are decided by communication of the objects [22].

LogSim: A parallel logic simulator for VLSI circuits to verify their logical and timing specifications. The program adopts a virtual time algorithm [23].

DisPool: A benchmark program for the distributed pool [24] which is a table maintenance utility that provides various forms of tables with arbitrary keys and data. As the distributed pool is used with many application programs, this benchmark program shows characteristic of a real application.

Pentomino: A program to find out all solutions of a $6 \times 10$ packing piece puzzle. The Multi-level dynamic load balancing scheme [25] is employed.

COMPILE: Distributed compilation of AYA language processor [26] which is written in KL1. The language processor consists of $21 \mathrm{KL} 1$ source program files, each of which is compiled on different processor.

\section{Comparison with Using Acknowledgements}

Termination can be detected using acknowledgements. Each processor has a counter which is incremented on sending a message, and is decremented on receiving an acknowledge message. When the value of the counter reaches zero, it is guaranteed that no messages are in transit [27].

We compare the number of additional messages of the two schemes. Under the scheme using acknowledgements, an acknowledge message is an additional 
Table 3. Frequency and cost of additional messages of the WTC scheme.

\begin{tabular}{|c|c|c|c|c|c|}
\hline Programs & $\begin{array}{r}\text { exec. time } \\
(\mathrm{sec})\end{array}$ & $\begin{array}{c}\text { additional } \\
\text { messages }\end{array}$ & $\begin{array}{l}\text { exec. } t \\
\text { (msec) }\end{array}$ & $\begin{array}{l}\mathrm{me} / \mathrm{msg} \\
\left(\mathrm{K} \text { red }^{\mathrm{a}}\right)\end{array}$ & 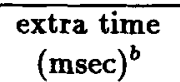 \\
\hline $32 \mathrm{PEs}$ & 1,986 & 119 & 16,689 & 10,013 & $6(0.0 \%)$ \\
\hline $256 \mathrm{PEs}$ & 348 & 868 & 401 & 240 & $43(0.0 \%)$ \\
\hline $32 \mathrm{PEs}$ & 5,457 & 3,164 & 1,725 & 1,035 & $158(0.0 \%)$ \\
\hline $256 \mathrm{PEs}$ & 793 & 25,982 & 31 & 19 & $1,299(0.2 \%)$ \\
\hline $64 \mathrm{PEs}$ & 104 & 1,343 & 77 & 46 & $67(0.0 \%)$ \\
\hline $256 \mathrm{PEs}$ & 356 & 767 & 464 & 278 & $38(0.0 \%)$ \\
\hline $32 \mathrm{PEs}$ & 53 & 1,119 & 47 & 28 & $56(0.1 \%)$ \\
\hline $256 \mathrm{PEs}$ & 249 & 7,851 & 32 & 19 & $393(0.2 \%)$ \\
\hline Pentomino 32 PEs & 665 & 1,163 & 572 & 343 & $58(0.0 \%)$ \\
\hline $256 \mathrm{PEs}$ & 134 & $13, \overline{331}$ & 10 & 6.0 & $667(0.5 \%)$ \\
\hline COMPILE 21 PEs & 282 & 810 & 348 & 209 & $41(0.0 \%)$ \\
\hline
\end{tabular}

a The performance is $600 \mathrm{KLIPS}$ in append on a PIM/m single processor.

${ }^{b}$ It takes the detecting process about $50 \mu \mathrm{sec}$ to handle each additional message.

one which is sent in response to \%throw goal or \%unify messages. Under the WTC scheme, \%terminated, \%request, and \%supply are additional messages.

Table 2 shows the number of the additional messages of the two schemes. As can be seen, except for Gene, the additional messages sent under the WTC scheme are almost negligible compared with the ones of the scheme using acknowledgements.

\section{The detecting process can be a bottleneck ?}

Under the WTC scheme, additional messages for termination detection are all sent or received by the detecting process. Therefore, if the additional messages are sent frequently, the detecting process becomes bottleneck.

The frequency and cost of sending or receiving additional messages are shown in table 3; the execution times per one additional message and the extra times consumed for handling additional messages are indicated. The execution times are also represented in append kiloreductions. The table demonstrates that the frequency is low enough and the extra time is negligible. Thus it can be concluded that the detecting process never becomes bottleneck.

\section{The postmortem predicate}

The postmortem predicate provides programmers with meta programming facilities. The form of the predicate is as follows.

postmortem (+GOAL, -RESULT) 
The predicate registers the postmortem goal specified by the GOAL, which is executed after detection of quiescence (termination or global suspension). As a postmortem goal has arbitrary arguments, it can access any data objects generated before quiescence detection through those arguments.

When registration is done, RESULT is unified with []. Waiting for the instantiation will prevent further processing from being executed before the completion of the registration. If this predicate is called more than once, the newly specified goal is appended to the old ones.

\section{Distributed Implementation}

Implementation of the postmortem predicate in distributed environments consists of the following three phases:

1. Memorizing the postmortem goals.

2. Detection of quiescence.

3. Execution of the memorized goals.

Operations on each phase are as follows.

Memorizing the postmortem goals: The processor where the postmortem predicate is executed memorizes the postmortem goals. If the processor already has postmortem goals memorized, it appends the new goal to the old ones.

Detection of quiescence: The detecting process detects quiescence using the scheme described in section 3.

Execution of the memorized goals: When the detecting process detects quiescence, it broadcasts a \%start message demanding execution of the postmortem goals memorized. A \%start message, like \%supply, also brings a large amount of weight to a destination processor. On receiving a \%start message, if postmortem goals reside in it, the processor adds the weight carried to its weight, and starts the execution of the goals. Otherwise, the \%start message is treated in the same way as the case of a \%supply message.

Note that a processor need not be concerned with quiescence. A processor which starts execution of postmortem goals can send a message at any time. Although the message may reach the other processor earlier than a \%start message does, the processor need not be concerned with the order.

\section{Summary}

Schemes for termination and global suspension detection in a distributed KLIC implementation are described.

Quiescence detection is fundamental to parallel and distributed processing. It is not easy to detect quiescence for distributed computation because of the difficulty in obtaining a consistent global state, especially when there can be messages in transit. We have solved the problems using the weighted throw counting technique. Experimental studies on a PIM/m machine have shown that the 
scheme is superior to the scheme using acknowledgements, and the detecting process never becomes a bottleneck.

The postmortem predicate which provide meta programming facilities for programmers was designed, and distributed implementation was done for the first time.

\section{References}

1. T. Chikayama, T. Fujise, and D. Sekita, "A Portable and Efficient Implementation of KL1," Proc. International Symposium on Programming Language Implementation and Logic Programming, LNCS 844, pp.25-39, 1994.

2. K. Ueda and T. Chikayama, "Design of the Kernel Language for the Parallel Inference Machine," The Computer Journal, Vol.33, No.6, pp.494-500, 1990.

3. K. Rokusawa, N. Ichiyoshi, T. Chikayama, and H. Nakashima, "An Efficient Termination Detection and Abortion Algorithm for Distributed Processing Systems," Proc. International Conference on Parallel Processing, Vol.I, pp.18-22, 1988.

4. K. Nakajima, Y. Inamura, N. Ichiyoshi, K. Rokusawa, and T. Chikayama, "Distributed Implementation of KL1 on the Multi-PSI/V2," Proc. International Conference on Logic Programming, pp.436-451, 1989.

5. K. Hirata, R. Yamamoto, A. Imai, H. Kawai, K. Hirano, T. Takagi, K. Taki, A. Nakase, and K. Rokusawa, "Parallel and Distributed Implementation of Concurrent Logic Programming Language KL1," Proc. International Conference on Fifth Generation Computer Systems 1992, pp.436-459, 1992.

6. K. Ueda, "Guarded Horn Clauses: A Parallel Logic Programming Language with the Concept of a Guard," Technical Report TR-208, ICOT, 1986.

7. E. Shapiro, "Systems Programming in Concurrent Prolog," Logic Programming and its Applications, M, van Canegham and D.H.D. Warren (eds.), Albex Publishing Co., pp.50-74, 1986.

8. K. Clark and S. Gregory, "PARLOG: Parallel Programming in Logic," ACM Transactions on Programming Languages and Systems, Vol.8, No.1, pp.1-49, 1986.

9. V. A. Saraswat, K. Kahn, and J. Levy, "Janus: A Step Towards Distributed Constraint Programming," Proc. North American Conference on Logic Programming, pp.431-446, 1990.

10. N. Ichiyoshi, K. Rokusawa, K. Nakajima, and Y. Inamura, "A New External Reference Management and Distributed Unification for KL1," New Generation Computing, Ohmsha Ltd., pp.159-177, 1990.

11. K. Rokusawa, A. Nakase, and T. Chikayama, "Distributed Memory Implementation of KLIC," Proc. Workshop on Parallel Logic Programming and its Programming Environments, Technical Report CIS-TR-94-04, University of Oregon, pp.151-162, March, 1994. Also New Generation Computing, Ohmsha Ltd., to appear.

12. P. Watson and I. Watson, "An Efficient Garbage Collection Scheme for Parallel Computer Architectures," Proc. Parallel Architectures and Languages Europe, LNCS 259, Vol.II, pp.432-443, 1987.

13. D. I. Bevan, "Distributed Garbage Collection Using Reference Counting," Parallel Computing, Vol.9, No.2, pp.179-192, 1989.

14. G. Tel and F. Mattern, "The Derivation of Distributed Termination Detection Algorithms from Garbage Collection Schemes," Proc. Parallel Architectures and Languages Europe, LNCS 505, Vol.I, pp.137-149, 1991. 
15. F. Mattern, "Global Quiescence Detection Based on Credit Distribution and Recovery," Inf. Proc. Lett., Vol.30, No.4, pp.195-200, 1989.

16. T. Chikayama and Y. Kimura, "Multiple Reference Management in Flat GHC," Proc. International Conference on Logic Programming, pp.276-293, 1987.

17. Y. Inamura, N. Ichiyoshi, K. Rokusawa, and K. Nakajima, "Optimization Techniques Using the MRB and Their Evaluation on the Multi-PSI/V2," Proc. North American Conference on Logic Programming, pp.907-921, 1989.

18. Y. Inamura and S. Onishi, "A Detection Algorithm of Perpetual Suspension in KL1," Proc. International Conference on Logic Programming, pp.18-30, 1990.

19. R. Hasegawa and M. Koshimura, "An AND Parallelization Method for MGTP and Its Evaluation," Proc. First International Symposium on Parallel Symbolic Computation, pp.194-203, 1994.

20. M. Fujita, J. Slaney, and F. Bennett, "Automatic Generation of Some Results in Finite Algebra," Proc. IJCAI-93, 1993.

21. M. Ishikawa, T. Toya, and Y. Totoki, "Parallel Application Systems in Genetic Information Processing," Proc. International Symposium on Fifth Generation Computer Systems 1994, pp.129-138, 1994.

22. H. Date and K. Taki, "A Parallel Lookahead Line Search Router with Automatic Ripup-and-reroute," Proc. EDAC-EUROASIC 93, 1993.

23. Y. Matsumoto and K. Taki, "Parallel Logic Simulator Base on Time Warp and Its Evaluation," Proc. International Conference on Fifth Generation Computer Systems 1992, pp.1198-1206, 1992.

24. M. Sato, M. Yamauchi, and T. Chikayama, "Distributed Pool and Its Implementation," Proc. International Symposium on Fifth Generation Computer Systems 1994, pp.90-99, 1994.

25. M. Furuichi, N. Ichiyoshi, and K. Taki, "A Multi-Level Load Balancing Scheme for OR-Parallel Exhaustive Search Programs on the Multi-PSI," Proc. Second ACM SIGPLAN Symposium on Principles and Practice of Parallel Programming, pp.50$59,1990$.

26. K. Susaki, et al., "Programming in KL1 and AYA," Technical Report TR-831, ICOT, 1993.

27. N. Shavit and N. Francez, "A New Approach to Detection of Locally Indicative Stability," Proc. International Colloquium on Automata, Languages and Programming, LNCS 226, pp.344-358, 1986. 